\title{
Fundamentos teóricos y metodológicos para un estudio lexicográfico del verbo asquear
}

\author{
José-Álvaro Porto Dapena \\ Universidade da Coruña
}

Desde el punto de vista semasiológico, que es el adoptado normalmente en la descripción lexicográfica, en el estudio de los verbos es, desde luego, primordial partir de sus características sintagmáticas. Es decir, antes de establecer sus respectivos significados o acepciones, deben determinarse sus valencias, actantes o argumentos, lo que, por otro lado, nos permitirá establecer su estructura argumental y, a partir de ella, el paradigma o paradigmas semánticos de que el verbo en cuestión forma parte. Pues, como ya señalé en alguna ocasión ${ }^{1}$, es evidente que un verbo al que corresponden diversas estructuras argumentales pertenecerá, sin lugar a dudas, en cada una de ellas a un paradigma léxico diferente. No se puede afirmar, sin embargo, lo contrario, pues un verbo, para pertenecer a paradigmas léxicos distintos, no siempre le es indispensable poseer estructuras actanciales diferentes: dicha pertenencia puede venir dada también por características extraargumentales correspondientes a otros aspectos discursivos, tanto contextuales como meramente pragmáticos.

En las líneas que siguen tendremos ocasión de observar todo esto en relación con el verbo asquear, cuyo estudio lexicográfico me propongo realizar aquí, como una contribución más al Diccionario "Coruña" de la lengua española actual (DCLEA), en cuya redacción nos hallamos empeñados ${ }^{2}$. He de destacar, además, que este verbo presenta unas especiales características sintáctico-semánticas que lo convierten en prototipo de lo que aquí voy a llamar verbo bifocal, esto es, con dos posibilidades adlativa y ablativa propias de parejas que, como comprar-vender o preguntar-responder, se hallan en una relación de inversión ${ }^{3}$.

1 Véase Porto Dapena (2002b).

2 Este pequeño trabajo se inscribe, pues, en la nómina de los realizados al amparo del proyecto de investigación HUM2004-05074/FILO, financiado por el Ministerio de Educación y Ciencia.

3 Sorprendentemente, este verbo no forma parte de la nómina o macroestructura del Diccionario de construcción y régimen de R. J. Cuervo. Ni siquiera yo mismo lo incluí -por descuido-en la lista suplementaria de entradas que para este diccionario elaboré en 1980 (véase Porto Dapena 1980: 349). 


\section{ESTRUCTURAACTANCIAL Y SINTÁCTICA}

Tratando, así pues, de establecer en primer lugar la estructura actancial de este verbo, cabe observar que tiene ante todo dos argumentos (A1 y A2), constituidos, respectivamente, por la persona que experimenta el asco (llamémosle experimentador) y aquello que lo produce, es decir, la causa. Tal estructura se manifestaría, por ejemplo, en un enunciado como (1):

(1) Enrique (A1) asqueó el pescado (A2).

Al lado de estos actantes o argumentos fundamentales puede aparecer un tercero (A3) e incluso, más raramente, un cuarto (A4), representados, respectivamente, por el órgano afectado, concretamente el estómago (podemos considerarlo como un locativo), y por una segunda persona que actúa como instigador, como ocurre, por ejemplo, en (2) y (3):

(2) El pescado (A2) le (A1) asqueó el estómago (A3).

(3) La señora de la pensión (A4) terminó por asquear a Enrique (A1) totalmente del pescado (A2).

Es necesario, por otro lado, en relación con la configuración sintáctica de estos argumentos hacer las siguientes observaciones:

a) Cuando no existen más que A1 y A2, éstos pueden intercambiar sus funciones sintácticas, actuando uno como sujeto y el otro como objeto directo, que solamente es obligatorio cuando éste es A2. Esto es precisamente lo que, como veremos, hace que el verbo sea bifocal. Es decir, además de (1), tenemos las posibilidades (4) y (5):

(4) El pescado (A2) asqueó a Enrique (A1)

(5) El pescado (A2) asquea

No sería posible

(6) Enrique asquea,

a menos que Enrique represente la causa, esto es, A2, es decir, en el sentido de que 'da asco'. Existe también la construcción pronominal, actuando A1 como sujeto y pasando $\mathrm{A} 2$ a complemento preposicional con de:

(7) Enrique (A1) se asqueó del pescado (A2).

b) En el caso -raro- de que exista A4, éste actúa siempre como sujeto, y a su lado A1 será objeto directo o implemento, mientras que A2, si se expresa, aparecerá como complemento preposicional con de (véase ej. 3). Y en el caso extremo de que se expresara $\mathrm{A} 3$, éste funcionaría obligatoriamente como implemento, pasando por su parte A1 a complemento u objeto indirecto (dativo posesivo más bien), y A2 aparecería como complemento preposicional. Así, 
(8) La señora (A4) de la pensión terminó por asquearle el estómago (A3) a Enrique (A1) con tanto pescado (A2).

c) Finalmente, notemos que A3, representado siempre por el sustantivo estómago, con el que, por consiguiente, el verbo presenta una solidaridad, actúa, en vez de A1, como sujeto o, también, como implemento. Por esa razón A1 tiene que funcionar siempre como complemento indirecto (dativo posesivo) o pasar a incluirse semánticamente en un adjetivo o complemento posesivo, mientras que A2 habrá de caracterizarse semánticamente por contener el rasgo semántico 'alimento' (habrá otra solidaridad en este caso) y actuará también, en alternancia con A3, como implemento o sujeto. Finalmente, A3 como sujeto admite también la construcción pronominal. Según esto, todavía existen estas otras dos posibilidades:

(9) El estómago (A3) de Enrique (A1) asqueó el pescado (A2).

(10) El estómago (A3) de Enrique (A1) se asqueó del pescado (A2).

\section{DETERMINANDO EL PARADIGMA O PARADIGMAS}

El hecho de que A2 y A3 sean objeto de sendas solidaridades por parte del verbo implica que éste debe de tener en su compañía un significado diferente del que posee en otros contextos, en que no aparecen; así, por ejemplo,

(11) Me (A1) asquea la politica (A2),

donde, por una parte, sería imposible la aparición de A3 (comp.

(12) * La política (A2) me (A1) asquea el estómago (A3) $)$,

y, por otra, A2 no cumple la condición semántica de ser un alimento.

El tipo de contextos en que puede aparecer A3 es, por tanto, particular, y el verbo tendrá que tener un contenido también especial, relacionado con la alimentación, pues, a la vez, se dirá de un alimento o se aplicará a él. Si acudimos a la palabra asco, de la que asquear es, obviamente, un desarrollo ${ }^{5}$, observaremos que posee los significados de 'alteración del estómago causada por la repugnancia que se tiene a algo que incita a vómito' al lado de 'impresión desagradable causada por algo que repugna', según las definiciones registradas en el $D R A E$. Es evidente, por

4 La frase podría utilizarse, no obstante, expresivamente, jugando con un doble sentido de asquear, al igual que, por ejemplo, podríamos decir "La política me da ganas de vomitar" en clara y gráfica alusión al asco que nos produce.

5 Se trata, como es obvio, de un verbo formado a partir del sustantivo asco, y que hace su aparición en nuestra lengua en el siglo XVI; según el CORDE, aparece por primera vez en este texto de M. de Chaide (1588): "Tienen otra cosa rara, digna de tales sujetos, y es que, si oyen algo fuera de lo que ellos han leído en cuatro autores de Gramática, lo asquean tanto y lo burlan y mofan de tal suerte, como si sólo aquello con que éllos han desayunado su entendimiento".

Sobre el concepto de 'desarrollo', véase Coseriu (1977: 138). 
tanto, que asquear en los contextos en que puede aparecer A3 y en que A2 representa un alimento tiene que referirse al asco como alteración estomacal, mientras que en los otros casos, como (11), aludirá a asco en su segunda acepción.

Esto significa que, si asco posee dos significados o acepciones -en el sentido estricto de invariantes semánticas- diferentes, también habrá de tenerlas su derivado asquear. Y así es en efecto: en el primer caso este verbo entrará en la órbita de, entre otros, indigestar, caer (bien o mal), revolver (el estómago o el cuerpo), empachar, intoxicar, envenenar, mientras que en el segundo corresponderá más bien al paradigma de repeler, rechazar, repugnar, odiar, etc. En apoyo de esta distinción, notemos que solo en este último caso asquear-aunque solo en su enfoque adlativo, como veremos- puede ser sustituido por la expresión perifrástica dar as$\mathrm{co}^{6}$, tal como se demuestra en

(13) Me asquea la política $=$ Me da asco la política.

frente a

(14) El pescado le asqueó el estómago $=*$ El pescado le dio asco al estómago.

que no admitiría la conmutación. La equivalencia, no obstante, parece que se da más bien en las formas imperfectivas, porque asquear en las perfectivas aporta normalmente un matiz resultativo -marca como el inicio de un estado, resultado de la acción-, que no pose dar asco; de ahí la diferencia entre:

(15a). Me asqueó la política (esto es, me dejó asqueado: el asqueamiento puede persistir todavía),

(15b) Me dio asco la política (me hizo sentir asco en un momento determinado).

\section{INCIDENCIA DE LOS ASPECTOS FÍSICO Y MORAL}

Cabe plantearnos ahora si en los casos en que el argumento A2 no incluye el rasgo semántico 'alimento' no convendrá hablar a su vez de la pertenencia de asquear a otros dos paradigmas léxico-semánticos diferentes, atendiendo al hecho de que este verbo aluda a un asco físico, esto es, al 'sentimiento de repugnancia que nos produce la percepción desagradable de un objeto a través de los sentidos', como sería el caso en

(16) Le (A1) asqueaba aquel olor (A2) a basura,

o a un asco moral, es decir, como 'sentimiento de repugnancia que en nuestro espíritu nos produce algo que nos molesta o alguien que no se atiene a nuestros principios éticos o morales'; tal es el caso de (11) o, asimismo, el de (17):

6 La razón es, obviamente, porque en esta frase asco se entiende en la segunda y nunca en la primera acepción: dar asco es producir esa sensación o sentimiento, no provocar una determinada reacción en el estómago. 
(17) Me (A1) asquea tener (A2) que ir a trabajar todos los días

En ambos casos es cierto que asquear se inscribe en dos universos de discurso diferentes, el físico y el espiritual o moral, que en múltiples ocasiones sirven de hecho para separar significados distintos de una misma palabra, o lo que es lo mismo, para adscribir ésta a sendos paradigmas léxico-semánticos. No es éste, sin embargo, a mi juicio, el caso del verbo que nos ocupa, puesto que en ambos tipos de contextos asquear -con independencia de que puedan a su vez darse matices más precisos- se sigue oponiendo a las mismas palabras y, por lo tanto, no cambia de paradigma léxico-semántico. Y así, tanto en (16) como (17) el verbo podría alternar, entre otros, con repugnar, dar asco, molestar, fastidiar, disgustar, hastiar, cansar, aburrir, etc. Digamos que no varía el significado, sino el puro sentido -recto o figurado- en que se toman los verbos en cuestión.

Notemos, por otra parte, que, hablando exclusivamente en sentido moral, tampoco responden a idéntico contenido expresiones como

(18) Nos asquea la hipocresía y la mentira,

(19) Me asquea que siempre me esté contando las mismas cosas.

En este último caso, efectivamente, el verbo asquear -o el sustantivo asco que le subayace- parece responder más bien a un uso hiperbólico, lo que hace a este verbo sinónimo funcional de cansar, hastiar o aburrir. Pero no se trata, obviamente, de una nueva acepción, sino de una mera variante contextual, consistente en la neutralización de asquear con esos verbos, que, en condiciones normales, presentan un contenido menos intenso que aquél ${ }^{7}$.

\section{ACEPCIONES Y SUBACEPCIONES, SEGÚN LOS DICCIONARIOS}

Pero todo esto nos lleva a plantearnos las acepciones, o invariantes, y subacepciones, o variantes, semánticas de este verbo, al que por cierto el $D R A E$ atribuye una sola acepción, susceptible -eso sí- de dos construcciones: una intransitiva y otra transitiva. Así,

asquear. intr. Dicho de una cosa: Causar asco. Este trabajo me asquea. U. t. c. tr.

De acuerdo con el ejemplo aducido, es obvio que el pronombre me, representante de A1, es interpretado por la Academia como un dativo o complemento indirecto, pues de otro modo no se explicaría la calificación de intransitivo que hace de este verbo, interpretación, a mi modo de ver, incierta, pues A1 funciona aquí como verdadero complemento directo o implemento, como lo demuestra el hecho de que

7 El único diccionario que registra, si bien como subacepción, esta posibilidad es el DUE de M. Moliner, quien por cierto la marca como perteneciente a un registro informal. 
el participio asqueado es perfectamente aplicable al experimentador; de ahí las posibilidades (16), (17) o (18):

(20) Este trabajo me tiene asqueado,

(21) Estoy asqueado por (o a causa de) este trabajo,

(22) Este trabajo me deja asqueado.

Como no lo explica suficientemente, no se sabe a qué tipo de construcción transitiva se refiere con la indicación "u. t. c. tr."

Más explícito, desde luego, es el DUE de M. Moliner, al distinguir dos acepciones (con una subacepción en la segunda), si bien una de ellas, la primera, de poco uso, a saber:

asquear. 1 intr. Sentir asco. 2 tr. Producir una cosa *repugnancia moral a \ alguien. $\simeq$ Dar asco. $\odot$ Aburrir o fastidiar una cosa, un trabajo, etc., a $\searrow$ alguien.

Es curioso que ahora la acepción 1 del $D R A E$, que aquí coincide con la 2, es calificada exclusivamente como transitiva, atribuyéndose, en cambio, carácter intransitivo a 1, que se correspondería, lógicamente, con la posibilidad que antes hemos apuntado en el ejemplo (6) (esto es, A1 + verbo) y que hemos considerado inviable sencillamente porque A1 como sujeto no puede aparecer sin A2 como objeto directo.

Por lo demás, la separación de acepciones parece basarla M. Moliner en los caracteres transitivo ( $2^{\mathrm{a}}$ acep.) e intransitivo ( $1^{\mathrm{a}}$ acep.), aunque, en el fondo, me inclino a pensar que parte más bien del hecho de que el sujeto sea A1 o A2, lo que, por otro lado, se corresponde, como sería de esperar, con una diferencia semántica evidente: si el sujeto es el experimentador, esto es, A1, asquear equivale a 'sentir asco', mientras que si lo es la causa (A2) equivaldrá a 'causar o producir asco'.

Solo por citar un diccionario más, el $D E A$ de M. Seco, O. de Andrés y G. Ramos es el más explícito de todos, al establecer tres acepciones distintas, divididas en dos grupos, transitivo (acep. 1) e intransitivo, y este último en normal (acep. 2) -o intransitivo propiamente dicho-y pronominal (acep. 3). Así,

asquear A $\operatorname{tr} 1$ Sentir o mostrar asco [por alguien o algo $(c d)$ ].

B intr > a normal 2 Causar asco [a alguien una pers. o cosa].

b > pr (?se) 3 Sentir asco [de algo]. Frec en part. Tb sin compl.

Es curioso señalar que precisamente la acepción transitiva coincida, incluso en la definición, con la 1 (intransitiva) del $D U E$ de M. Moliner, y que, por otro lado, la acepción 2, intransitiva normal, coincidente con la 1 del $D R A E$, se defina como

Causar asco [a alguien una persona o cosa]

donde a alguien deberá interpretarse, aunque no se especifique la función, como objeto indirecto o dativo. Pero lo más llamativo es la coincidencia semántica entre 
las acepciones 1 y 3 , que se diferenciarán exclusivamente por el tipo de construcción, transitivo en 1 e intransitivo pronominal en 3.

En conclusión, los criterios de separación de acepciones utilizados por estos tres diccionarios atienden en el caso de asquear al aspecto puramente sintáctico, procedimiento que sería correcto si las distinciones sintácticas fuesen siempre acompañadas de diferencias semánticas, cosa evidentemente falsa, como lo demuestran, por ejemplo, las acepciones 1 y 3 del $D E A$. El criterio semántico, que es el que con propiedad debe utilizarse, se pasa totalmente por alto; es decir, no se atiende para nada al hecho de si asquear pertenece o no en sus diversos usos a paradigmas léxicos distintos, cosa que realmente ocurre, como hemos visto, cuando F2 es un alimento en relación con los casos en que no lo es. Por eso los diccionarios aludidos no registran -ni siquiera en subacepción especial- lo que sin duda constituye una auténtica acepción y que podría definirse como 'alterar el estómago' frente a 'causar o sentir asco', que es en definitiva el único significado recogido por estos diccionarios.

\section{BIFOCALIDAD Y ACEPCIONES}

Una característica sin duda fundamental del verbo asquear es su bifocalidad, es decir, la posibilidad, ya apuntada anteriormente, de poderse enfocar tanto desde F2 como desde F1 o, incluso, F3, tomados como sujeto. Es decir,

(23) Le asquean los niños impertinentes (A2) / Él (A1) asquea los niños impertinentes,

(24) La cerveza (A2) me asqueó el estómago / Mi estómago (F3) asqueó la cerveza / Yo (A1) asqué la cerveza.

La situación es muy semejante a la que se produce en otros verbos, como, por ejemplo, alquilar, que, precisamente por su bifocalidad, puede resultar ambiguo en enunciados como

(25) Le alquilé un piso a Gregorio

porque el sujeto -y lo mismo el complemento- puede ser tanto el propietario del piso (A1) como el usufructuario (A2): en el primer caso, efectivamente, alquilar viene a equivaler a 'dejar en alquiler', mientras que en el segundo, a 'tomar en alquiler'.

La bifocalidad es relativamente frecuente en verbos que son susceptibles de usarse como transitivos e intransitivos, pasando en este último caso a sujeto el implemento de la construcción transitiva. Es lo que ocurre, por ejemplo, en

(26) El disgusto enfermó a Juan / Juan enfermó con el disgusto

(27) El médico cura a los enfermos / Los enfermos curan 
(28) La muchacha huele la flor / La flor huele muy bien.

Todavía más frecuentemente se produce la bifocalidad en un mismo verbo por medios gramaticales, esto es, convirtiéndolo en pronominal o, claro está, construyéndolo en pasiva ${ }^{8}$ :

(29) Rogelio cerró la puerta / La puerta se cerró

(30) Alguien difundió la noticia / La noticia fue difundida / Se difundió la noticia.

Lo que, sin embargo, resulta harto infrecuente es que esa bifocalidad se produzca, como ocurre en asquear, con un intercambio mutuo de funciones entre sus actantes, esto es, pasando, por una parte, el objeto a sujeto, y, por otra, el sujeto a objeto, tal como sucede, por ejemplo, en (23). Hablamos entonces de bifocalidad inversa, porque el verbo presentará, correlativamente, dos contenidos semánticos inversos, comparables a los de parejas en relación de inversión como comprar/vender, ir/venir, subir/bajar, dar/recibir, preguntar/responder, etc.

En el caso que nos ocupa es evidente que, cuando el sujeto es A1, el verbo asquear equivale a 'sentir asco', mientras que cuando lo es A2, la equivalencia semántica es otra: 'causar o producir asco'. Hay, por lo tanto, una evidente diferencia semántica entre una y otra configuración sintáctica, circunstancia que fácilmente podría llevarnos - tal es el caso, como vimos, del $D U E$ y también del $D E A$ - a postular la existencia de dos acepciones diferentes. Ello vendría, además, avalado por el hecho de que en un caso asquear tiene a sentir como incluyente lógico en su definición, mientras que este papel corresponde en el otro a causar o producir, incluyentes ambos que, por otra parte, no pertenecen, como es obvio, al mismo paradigma léxico-semántico. De hecho en el primer caso asquear se opone a verbos como rechazar, odiar o repugnar, mientras que en el otro se relacionará más bien con cansar, hastiar o molestar.

Notemos, sin embargo, que los incluyentes sentir y causar, que bien podrían figurar en las respectivas definiciones lexicográficas -constituidas entonces por expresiones endocéntricas- no son, como sería de esperar, verdaderos hiperónimos de asquear y, por lo tanto, las definiciones así formuladas no serán -contra lo que pudiera parecer- auténticas definiciones hiperonímicas. Tengamos en cuenta que, si realmente se diera esa relación hiperonímica entre estos verbos y el definido, nuestro verbo formaría parte en ambos casos de sendas listas interminables de palabras; por ejemplo, solo bajo el hiperónimo sentir, tendríamos, entre otros, compadecer, agradecer, almadiar, apreciar, amar, acerar, carraspear con un larguísimo etcétera.

8 La bifocalidad constituye, efectivamente, como ya señaló B. García Hernández (1980 y 1998), una especie de diátesis, pero producida en el nivel exclusivamente léxico; distinta, por lo tanto, a la que se produce por medios morfológicos o incluso sintácticos, como es el caso de la pronominalización de ciertos verbos transitivos o la distinción de voz activa y pasiva. 
Como pienso poner de manifiesto en un futuro artículo sobre este tipo especial de definiciones -las que yo llamaré relacionales endocéntricas-, los verbos sentir y causar, aunque son núcleos sintácticos del sintagma definicional, no juegan a su vez en este caso, al contrario de lo que ocurre en las hiperonímicas, el papel de núcleo semántico ${ }^{9}$. Este corresponderá a $a s c o^{10}$, palabra que por no coincidir con la categoría léxico-gramatical del definido necesita de un verbo soporte -en este caso sentir o causar - que actúa como una especie de transpositor (convierte, por así decirlo, ese sustantivo en verbo), aportando al mismo tiempo en el análisis semántico de la definición un contenido muy general, que en este caso atañe exclusivamente a la clase del verbo. Éste, efectivamente, queda con causar incluido en la clase que Coseriu denomina adlativa, en tanto que con sentir se adscribe a la ablativa. Podría también pensarse en una clase causativa o factitiva frente a otra no causativa; pero ésta distinción funciona propiamente tan solo en expresiones con A4. Así, por ejemplo, en el siguiente enunciado:

(31) El profesor asqueó a sus alumnos de las Matemáticas = El profesor hizo a sus alumnos asquear las Matemáticas.

No sería, en cambio, posible esta interpretación en

(32) Las Matemáticas asquean a los alumnos $=*$ Las Matemáticas hacen a los alumnos asquearlas

porque la causa tendría que desempeñar a la vez las funciones de sujeto y objeto directo.

En conclusión, pues, las diferencias semánticas que asquear presenta entre su contrucción adlativa (con A2 como sujeto) y la ablativa (esto es, con A1 con esa misma función) no implican la pertenencia de este verbo a paradigmas léxico-semánticos distintos. Por el contrario, se trata de un mismo paradigma, aunque -eso sí- escindido en dos clases verbales distintas y entre las que establece, como queda dicho, una relación de inversión.

\section{PROPUESTA DE ARTÍCULO LEXICOGRÁFICO}

Visto todo lo anterior, me parece justificado proponer para el DCLEA la siguiente redacción del artículo asquear, aprovechando así la ocasión para ofrecer aquí una pequeñísima muestra de cómo será este diccionario:

9 Entiendo por núcleo semántico de una definición lexicográfica la palabra que ostenta la mayor carga semántica y, por lo tanto, que aporta el contenido fundamental de la palabra definida. Contra lo que he asumido en otro lugar (véase Porto 2002: 292), no toda definición endocéntrica de incluyente positivo es identificable con la hiperonímica: ésta no es más que un tipo de aquélla, cuando el núcleo semántico coincide con el sintáctico.

10 Esta consideración coincide por cierto con la constitución morfológica de asquear, palabra formada, como ya se ha dicho, a partir del sustantivo asco, que, por lo tanto, constituye el núcleo fundamental o base de la derivación o, mejor, dicho, desarrollo 
asquear. $v$. Para la separación de aceps. partimos de dos conceptos de 'asco' diferentes: como afección estomacal (acep. 1) y como sensación o sentimiento (acep 2); bien es verdad que en ambos casos subyace la misma idea de 'rechazo' que podría dificultar el establecimiento de límites entre los respectivos paradigmas; obsérvese, no obstante, que solo en 2 este verbo equivale a dar asco. Notemos, por otro lado, que este verbo en cualquiera de esas aceps. admite una visión adlativa (Algo asquea a alguien), esto es, a partir de lo que causa o es motivo de asco, al lado de la ablativa (Alguien asquea algo o Alguien se asquea de algo) o a partir de quien experimenta ese asco. Por otro lado, en cada una de esas visiones el verbo se opone a conjuntos distintos de palabras, lo que podría hacer pensar en paradigmas también diferentes; no lo creemos así, pues de lo que en realidad se trata es de una división del paradigma en dos clases semánticas diferentes, adlativa y ablativa, a las que este verbo, como acabamos de ver, es indiferente, al incluir las dos posibilidades.

1. a) tr. [ una comida o bebida (suj.) el estómago (od.) < a alguien> (oi.)]. Dejárselo alterado de modo que sienta náuseas y repugnancia a recibir de nuevo ese u otro alimento: "Los callos me asquearon el estómago".

Que una proposición constructiva, un proyecto de beneficio social, un designio equis sea pasado por un arco del triunfo ya es bastante decepcionante; por media docena de arcos del triunfo, de forma consecutiva y despiadada, es para asquear al más encallecido estómago. (www.lahora.com.gt/04/06/17/paginas/ opinion.htm).

El obj. indirecto puede actuar también como directo: "Los callos me asquearon".

Nos levantábamos de madrugada para cazar palomas y tener así de comer. Luca hacía paté de hígado de paloma, que al principio estaba bien, pero después te asqueaba. (Pollimeni, Luca, 1991 (Argentina)). El café con leche y con azúcar lo asqueó. (Martini, El fantasma imperfecto, Alfaguara, 1994).

- prnl. Pasando a suj. el estómago o la persona afectada, 'sentir náuseas o repugnancia hacia un alimento por haber sentado mal': "Se me asqueó el estómago". Lo que produce el asco se expresa mediante de:

Intentó dos veces cambiarse al licor por instancias de la Gata; sin embargo, no pudo. Toda aquella extraña sensación de náusea en el estómago no valía la pena. Si se va a asquear uno de algo, se decía, que sea una patada al cerebro y no al estómago. (Obando Bolaños, El más violento paraíso, 2001). Se rieron todos juntos de los delirios del barbudo, se asquearon de los alimentos que ingerían en el barco y de la locura de lanzarse al mar en embarcación tan poco apropiada. (Santos Febres, Pez de vidrio y otros cuentos, 1996 (P. Rico)).

Se usa tal vez más en participio: “Tengo asqueado el estómago”, "Estoy asqueado".

b) tr. [ alguien (suj.) una comida o bebida (od.)]. Aburrirla, sentir repugnancia o rechazo hacia ella por haber sentado mal al estómago: "He asqueado los callos". En este caso el verbo adquiere carácter perfectivo, 
por lo que se usa preferentemente en formas a su vez perfectivas.

De regreso a Borgo San Sepolcro, a los pocos días comenzó a sudar el signor Capovilla y a quejarse de un punto fijo en la nuca, asqueó el cordero con arroz y los huevos hilados, pidió confesión, y pasó al otro mundo con un quejido. (Cunqueiro, Vida y fugas de Fanto Fantini della Gherardesca, Barcelona, 1972, p. 43).

Frecuente también en part. con tener: "Tengo asqueado el vino".

2. a) tr. [ una persona, animal o cosa (suj.) a otra persona (od.)]. Darle asco, producirle repulsión o aversión. Puede entenderse en un sentido físico, por resultar desagradable a los sentidos, o bien intelectual o moral; así, en sentido físico:

En el fondo, no le importa, aunque el olor a algas frescas a veces lo asquea y sale del restaurante o senso-club sosteniéndose la nariz. (Obando Bolaños, El más violento paraíso, S. José de C. Rica, 2001). El olor del cuerpo de su hermano, que le llegaba en vaharadas con el más mínimo movimiento que hacía, le asqueaba tanto como el suyo propio. (Chacón, La voz dormida, Madrid, 2002). Me asquea toda esa basura. (Caballero Bonald, Toda la noche oyeron pasar pájaros, Barcelona, 1988).

$\mathrm{Y}$ en sentido moral o intelectual:

No es que yo sea locamente sensible, pero llegó un momento en que la propia relación física me llegó a asquear. (Guelbenzu, El río de la luna, 1981). Como vecino de Moratalaz, me asquea tanto osado e ignorante atrevimiento, y quiero romper una lanza en favor del traslado del centro de Marroquina, dejando constancia de mi solidaridad con los afectados. (El Mundo, 27/12/1995: Pedro Oliva: El derecho a rehabilitarse). Como guatemalteco que soy, me sigue asqueando que un técnico extranjero venga recibir increíbles remuneraciones lo que a los nuestros les negamos. (La Hora, 22/05/1997 (Guatemala)).

- En este último sentido se usa hiperbólicamente equivaliendo a 'cansar o hastiar': "Me asquea tanto trabajar".

De la juventud no se ocupa nadie. Pero la juventud sólo es un trámite hacia otra cosa, y estos chicos tienen complejo de Peter Pan, no quieren crecer, al mismo tiempo que les asquea ser jóvenes. (El Mundo, 25/05/1995: Francisco Umbral: Kronen y movida).

- En construcción absoluta puede equivaler a, 'ser o resultar asqueroso'.

Podrá hacerse gran celebración de los mensajes televisivos usados y compartidos por millones de habitantes, pero, al fin, tanto personal asquea. (El País, 01/10/1986). Es que sois de un presumido que asquea... Yo no necesito pretextos, y si quiero estar con un tío, se lo digo y en paz. (Resino, Pop. y patatas fricas, 1992).

- prnl. Pasando a suj. el obj. directo y el suj., si se expresa, a compl. con de, 'sentir repulsión hacia algo sucio o desagradable':

A Sandra le huele el aliento, Sandra es la mujer de Nickie Marrana, le hiede el aliento y no se puede quitar la peste ni con medicinas, eso del mal olor es una desgracia que da asco y también risa, ¡hueles a cloaca, hueles a muerto!, en cambio hay otras desgracias 
que dan compasión y la gente ni se asquea ni se ríe. (Cela, Cristo versus Arizona, Barcelona, 1993). El cocalero Evo Morales se asquea de la política. (Los Tiempos, 03/02/1997 (Bolivia)).

Se usa quizá más en participio.

Estoy asqueado de la clase política. (Los Tiempos, 03/02/1997 (Bolivia)).

b) tr. [ alguien (suj) a otra persona (od.) <de algo $>$ (cp.)]. En este contexto, con un nuevo actante adquiere el sentido factitivo de 'hacer que otra persona se asquee de algo'.

Gaspard recurre a todo lo imaginable para encender la pasión, incluso a someterse a una disciplina de bata mugrienta, dentadura postiza, eructos y televisión para asquear a Camille del futuro que le espera si no pone remedio. (ABC, 19/08/1989). El gran mérito de Pasolini consiste en confundir, aburrir, exasperar, escandalizar y asquear a sus espectadores de modo que éstos salen de la sala mucho más conformes con sus pequeñas vidas, pero normales al fin. (López Navarro, Clásicos del cine, 1996).

c) tr. [ alguien (suj.) a una persona o cosa (od.)]. Sentir asco o repulsión hacia ella: "Asqueo las ratas".

¡Amo, perdóname! ¡Y que esto no te haga asquear a las mujeres! (Cunqueiro, Las mocedades de Ulises, Barcelona, 1970, p. 73).

\section{I asco, asqueamiento, asquerosidad, asqueroso.}

Como puede observarse, el artículo comienza por una pequeña introducción o "babero", donde se exponen brevemente cuestiones que atañen a la palabra estudiada de un modo general; concretamente se trata de justificar la separación que se hace de acepciones, así como poner de manifiesto la pertenencia de este verbo a las clases adlativa y ablativa.

Las acepciones, que se hallan indicadas con un número arábigo, están constituidas por varias subacepciones, las cuales se introducen mediante una letra minúscula con paréntesis de cierre. A su vez, la subacepciones pueden presentar variantes de uso, las cuales aparecen indicadas mediante el signo $\bullet$.

Cada acepción o subacepción comienza por una indicación entre corchetes donde se establece el contexto semántico (en una de sus configuraciones sintácticas) en que la palabra asquear posee el significado que se define a continuación. Los elementos que en dicho contexto aparecen entre paréntesis angulares $<>$ no son imprescindibles y, por lo tanto, pueden aparecer elípticos. Por su parte las palabras que en la definición se indican en cursiva corresponden al contorno, en coincidencia, por tanto, con elementos ya incluidos en el contexto, pero que aquí reaparecen por exigirlo la sintaxis del enunciado definicional.

Hay dos tipos de ejemplos: los propiamente dichos, que aparecen entre comillas y pertenecen a mi cosecha, junto a los registrados como autoridades. Estos últimos 
están tomados en su mayoría del $C R E A$, algunos del CORDE y, finalmente, otros de Internet utilizando el buscador Google, procedimento este último que se ha empleado cuando los córpora de la Academia carecían del material necesario.

Dado que aquí nos hemos ocupado más bien del aspecto semasiológico o, si se prefiere, sintagmático, he prescindido en el presente artículo lexicográfico del aspecto onomasiológico o paradigmático, que en su día tendrá también cabida en el $D C L E A$, donde se remitirá después de cada acepción al paradigma léxico-semántico correspondiente, contenido en otra parte de la obra. Se incluye, sin embargo, el paradigma morfológico, consistente en la lista de palabras emparentadas morfológicamente con la que hace de entrada y que se colocan al final del artículo precedidas por un calderón ( $($ ).

\section{REFERENCIAS BIBLIOGRÁFICAS}

CoSERIU, E. (1977): Principios de semántica estructural, Madrid, Gredos.

$[D E A]=$ SECO, M., O DE ANDRÉS y G. RAMos (1999): Diccionario del español actual, Madrid, Aguilar.

$[D R A E]=$ ReAl ACADEMIA EsPañola $\left(2001^{22}\right)$ : Diccionario de la lengua española, Madrid, Espasa-Calpe.

[DUE] $=$ Moliner, M. (19982): Diccionario de uso del español, Madrid, Gredos.

GARCíA HeRnÁNDEz, B. (1980): Semántica estructural y lexemática del verbo, Reus, Ediciones Avesta.

- (1998): "Clases semánticas y modificación prefijal en la estructura de campo", en G. Wotjak, coord, Teoría del campo y semántica léxica, Frankfurt am Main, Peter Lang, pp. 29-48.

Porto Dapena, J. A. (1980): Elementos de Lexicografía: El Diccionario de construcción y régimen de R. J. Cuervo, Bogotá, Instituto Caro y Cuervo.

- (2002a): Manual de técnica lexicográfica, Madrid, Arco/Libros.

(2002b): "La estructura actancial como criterio separador de acepciones en el artículo lexicográfico", en I. Pérez Pascual y M. Campos Souto, eds., Cuestiones de Lexicografia, Lugo, TrisTram, pp. 171-190. 